\title{
Motor imagery on upper extremity function for persons with stroke: a systematic review and meta-analysis
}

\author{
Dongsu Lee ${ }^{a}$, Sujin Hwang ${ }^{b}$ \\ ${ }^{a}$ Department of Physical Therapy, Graduate School, Baekseok University, Cheonan, Republic of Korea \\ ${ }^{b}$ Department of Physical Therapy, Division of Health Science, Baekseok University, Cheonan, Republic of Korea
}

\begin{abstract}
Objective: The purpose of this review was to investigate whether motor imagery training has an effect on the recovery of upper extremity function in individuals with hemiparetic stroke or not.

Design: A systematic review and meta-analysis.

Methods: PubMed and three other databases were searched up to December 18th, 2018 and randomized controlled trials (RCTs) evaluating motor imagery training on upper extremity function in persons with a diagnosis of hemiparetic stroke were included. This review selected the following information from each study: surname of the first author, published year, nation, population, intervention, therapeutic intensity of intervention, therapeutic comparison, outcome measures, additional therapy, summary of results, and descriptive statistics of outcome measures.

Results: This review selected seventeen RCTs with 487 stroke survivors and the following intervention methods: six motor imagery training with additional therapeutic technology, two motor imagery training with additional modified constraint-induced therapy, four mirror therapy, and five motor imagery training. Ten RCTs were eligible for meta-analysis after systematic review. The motor imagery group were more effective than the control group based on the Fugl-Meyer assessment (3.43; 95\% confidence interval $[\mathrm{CI}], 1.65$ to 5.22 ; heterogeneity $\left[\mathrm{chi}^{2}=8.03, \mathrm{df}=8, \mathrm{I}^{2}=0 \%\right.$ ], test of overall effect $\mathrm{Z}=3.76$; test for subgroup differences $\left.\left[\mathrm{chi}^{2}=2.56, \mathrm{df}=2, \mathrm{I}^{2}=21.8 \%\right]\right)$ and the Action Research Arm Test $\left(1.32 ; 95 \% \mathrm{CI},-8.12\right.$ to 10.76 ; heterogeneity $\left[\mathrm{Tau}^{2}=70.74\right.$, $\left.\mathrm{chi}^{2}=15.22, \mathrm{df}=3, \mathrm{I}^{2}=80 \%\right]$, test of overall effect $\mathrm{Z}=3.76$ ).
\end{abstract}

Conclusions: The results of this review suggests that motor imagery shows positive effectiveness on improving upper extremity function in persons with hemiparetic stroke.

Key Words: Imagery, Stroke, Upper extremity

\section{Introduction}

Ninety percent of stroke survivors suffer from persistent neurological motor deficits leading to disabilities and dysfunction caused by muscle weakness, loss of manual dexterity, muscle stiffness, pain, somatosensory impairments, visual impairments, spasticity, muscle contracture, and so on [1]. Among stroke survivors, recovery of function in the hemiparetic upper extremity has been noted to be fewer than $15 \%$ [2]. Stroke survivors often compensate for their paretic upper extremity by using their intact limb in the perform- ance of daily activities [3]. The compensation for the paretic upper limb can lead to the learned non-use phenomenon. Bimanual activities of both hands or unilateral activities of the paretic upper limb from the beginning of rehabilitation should be emphasized to gain optimal recovery and to prevent the learned non-use phenomenon.

Motor imagery is a representation of a movement in working memory without motor output, and is a multidimensional process that includes sensation, perception, attention, cognition, planning and programming, visuospatial reasoning, and memory [4-6]. Certain brain areas are acti-

Received: 24 February, 2019 Revised: 19 March, 2019 Accepted: 21 March, 2019

Corresponding author: Sujin Hwang (ORCID https://orcid.org/0000-0001-8471-0103)

Department of Physical Therapy, Division of Health Science, Baekseok University, 76 Munam-ro, Dongnam-gu, Cheonan 31065 , Republic of Korea Tel: 82-41-550-2309 Fax: 82-41-550-2829 E-mail: sujin928@gmail.com

(c) This is an Open-Access article distributed under the terms of the Creative Commons Attribution Non-Commercial License (http://creativecommons.org/licenses/ by-nc/4.0) which permits unrestricted non-commercial use, distribution, and reproduction in any medium, provided the original work is properly cited.

Copyright (๑) 2019 Korean Academy of Physical Therapy Rehabilitation Science 
vated at the beginning of the motor programming when imagining a physical movement. The neural circuits involve the primary motor cortex, the supplementary motor area, the premotor area, and the inferior parietal cortex [7]. Several studies have been conducted on motor imagery training to improve the paretic upper limb function and independent daily activities for persons with stroke $[4,6,8,9]$. Motor imagery training has been provided in a variety of ways, including motor imagery only, motor imagery with repetitive task-oriented approaches, motor imagery with electrical stimulation, and motor imagery with brain-computer interface in order to overcome the limitations following a stroke event [4,10-14].

This review was conducted to determine the effectiveness of motor imagery on the recovery of upper limb function compared to other therapeutic methods in persons who have had a stroke regardless of training types. The heterogeneity of the intervention types from the motor imagery only and motor imagery with brain computer interface intervention techniques used in order to improve the upper limb function of stroke survivors was also investigated.

\section{Methods}

The review was conducted in line with the Preferred Reporting Items for Systematic Reviews and Meta-Analyses (PRISMA) Statement for systematic review and meta-analysis. The search strategy of the review was performed by two researchers. To appraise and synthesize research-based evidence of the motor imagery on upper extremity function for stroke survivors, this review was according to the patient/participants/population/problem, intervention, comparison, outcome with timing, setting and study design.

\section{Search strategy}

The review protocol registered on PROSPERO (registration number: CRD42018109339) was based on the PRISMA. This review was conducted in accordance with the checklist of the PRISMA. The review was conducted from randomized controlled trials (RCTs) published up to December 18th, 2018 found through four academic electronic databases, including PubMed, EMbase, ProQuest, and EBSCO. The search strategy was a combination of the following MeSH terms and related terms: Stroke or CVA or brain vascular accident, Upper Extremity or Upper Limb, Imagery, and Randomized Controlled Trials or Randomized Clinical Trials. The search was restricted to studies in humans and those written in English only. In addition, the reference lists of all identified relevant publications were reviewed.

\section{Screening and eligibility of the selected studies}

After searching the studies through four databases and summing up the searched studies, we removed duplicate studies used on the title lists of selected studies. According to the topics of stroke, motor imagery, and upper extremity, two authors independently reviewed and determined whether the selected studies were acceptable or not. The studies were independently screened to see if they had met the inclusion criteria based on their titles and abstracts. The inclusion criteria were as follows: (1) the participants had a primary diagnosis of stroke without other neurological diseases; (2) RCTs that evaluated the effect of motor imagery on upper extremity function with the inclusion of a control group; (3) the study was written in English only; (4) human studies; and (5) the study was published as the full reports only. To be considered as a meta-analysis, studies were excluded if they did not include data that allowed the calculation of standard errors for effect estimates on motor imagery training for stroke.

\section{Collected data}

In the systematic review, the review extracted the surname of the first author, year, country and therapeutic setting, number of participations, intervention type, dosage and frequency of the interventions, comparison, outcome measures, additional therapy and summary of results. To analyze the risk of bias, we collected studies with random sequence generation, allocation concealment, blinding of patients, blinding of outcome assessment, incomplete outcome data, and selective reporting. We also extracted the mean and standard deviation of each outcome measure to conduct a meta-analysis.

\section{Data analysis}

The review was analyzed using the RevMan 5.3 (http:// ims.cochrane.org/revman) program. The risk of bias was analyzed (selection bias, performance bias, detection bias, attrition bias, and reporting bias). To assess the effect estimate of the selected RCTs, the mean and standard deviation values were pooled to obtain the mean difference and $95 \%$ confidence intervals (CI). The Higgins $\mathrm{I}^{2}$-statistic was performed to examine for heterogeneity. Heterogeneity of the pooled RCTs was divided into low level ( $\mathrm{I}^{2}$ of $25 \%$ to $50 \%$ ), moderate level ( $\mathrm{I}^{2}$ of values of $50 \%$ to $75 \%$ ), and high level 
( $>75 \%$ ). This review used fixed-effect models if the $\mathrm{I}^{2}$ test was not significant ( $p$ for heterogeneity $<0.1$ ), but random effect models used were significantly different.

\section{Results}

\section{Literature search and characteristics of the included RCTs}

Based on the initial search strategies, the review retrieved a total of 434 RCTs from four mainstream electronic databases but 354 records were removed due to duplication. Subsequently, 299 articles of the 354 were excluded based on the titles and abstracts, and 55 RCTs full-text articles were assessed for eligibility for the following reasons: 231 were non-RCTs, 33 were systematic reviews, 27 were grey literature, 5 were cross-over studies, and 3 studies were nonEnglish. Then, 38 full-text articles of the 55 were excluded for the following reasons; 9 were Quasi-RCTs, 20 did not have relevant outcomes, and 9 articles did not have therapeutic effects (Figure 1).

\section{Motor imagery training on upper extremity function for stroke survivors}

This review selected seventeen RCTs with 487 persons with hemiparetic stroke. The selected studies involved three different intervention types including motor imagery with addition of another intervention, motor imagery only, and mirror therapy. To assess the therapeutic effects of motor imagery training, the involved articles used variable outcome measures, such as the Fugl-Meyer assessment for upper extremity function (FMA-UE), the Action Research Arm Test (ARAT), Fugl-Meyer Assessment-motor recovery, Motor Activity Log, Jebson-Tayor Hand Function Test, Wolf Motor Function Test, stroke recovery (Brunnstrom's recovery stage, Modified Ashworth Scale, Stroke Impact Scale), activities of daily living (Functional Independent Measure, modified Barthel Index), Star Cancellation Test, motorevoked potentials, and positron emission tomography. All selected studies reported the positive effects of motor imagery training compared to the control group treatment except for only one study (Table 1).

\section{Risk of bias in all included RCTs}

All involved RCTs showed low risk in random sequence generation, allocation concealment, incomplete outcome data, and selective reporting. Nine studies showed a high risk of bias due to having a single-blinded study design [4,6,15-21]. One study showed a low bias risk in selection, performance, detection, attrition, and reporting bias [22]. Seven studies did not mention blinding of participations and personnel and so there were insufficient information to permit the judgement of whether they had a 'low risk' or 'high risk' of bias [5,8,9,23-26]. Four studies did not address

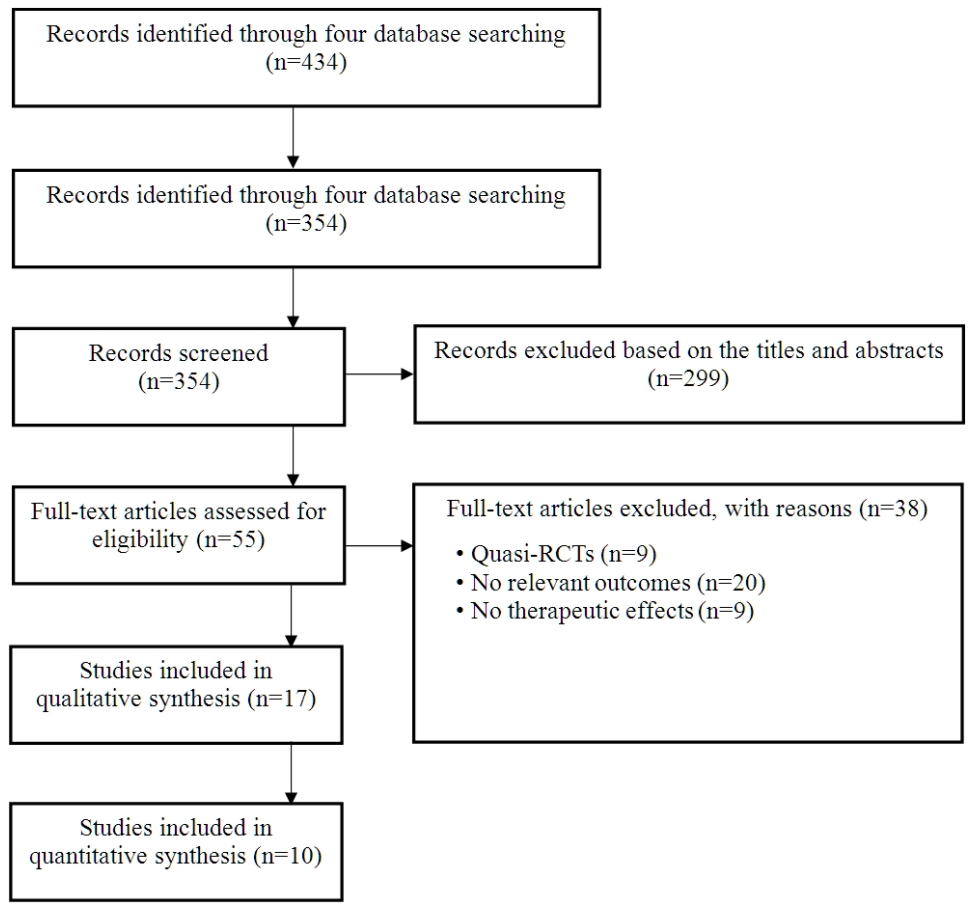

Figure 1. Flow diagram of included studies in the review. RCT: randomized controlled trial. 


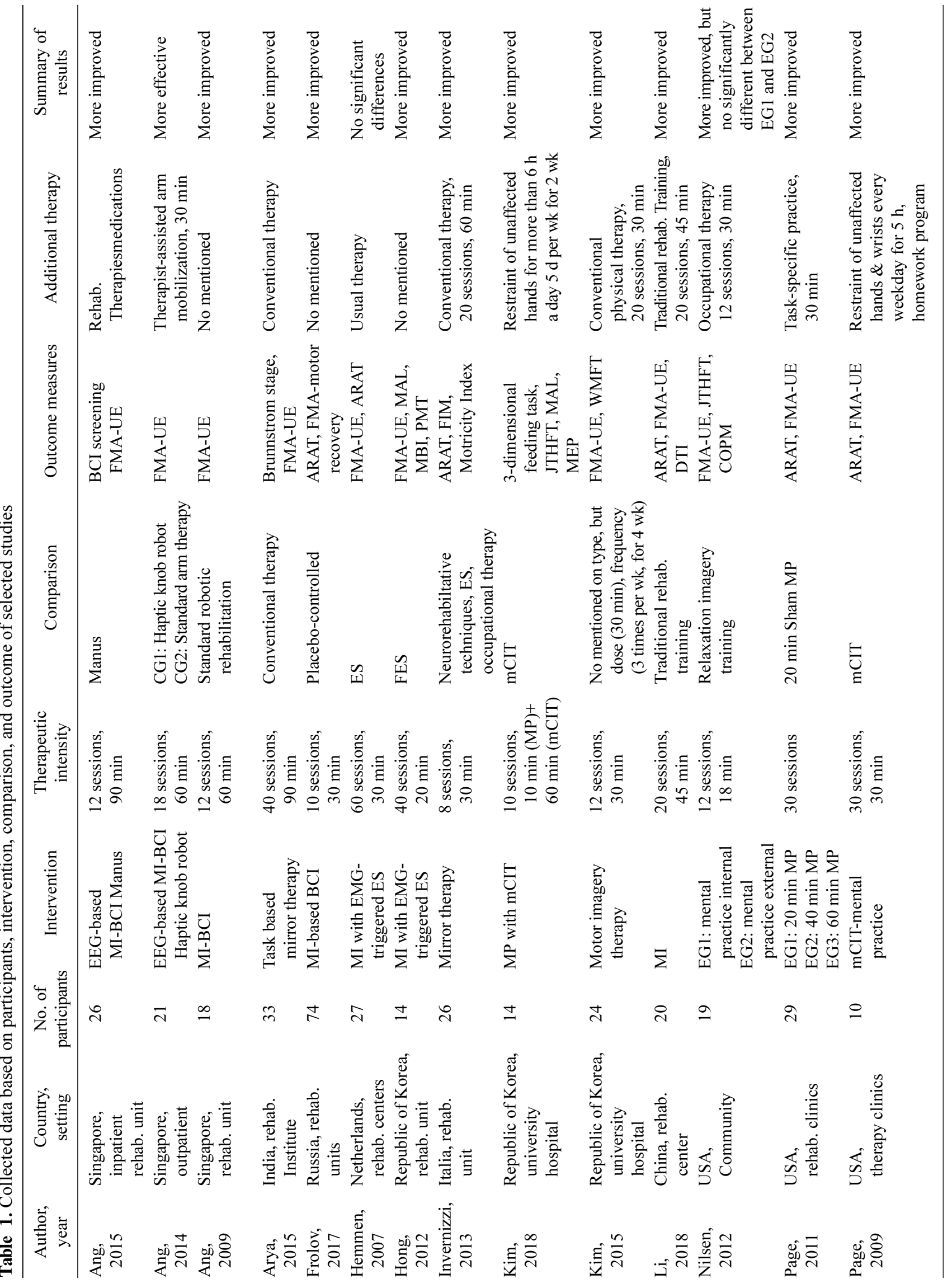




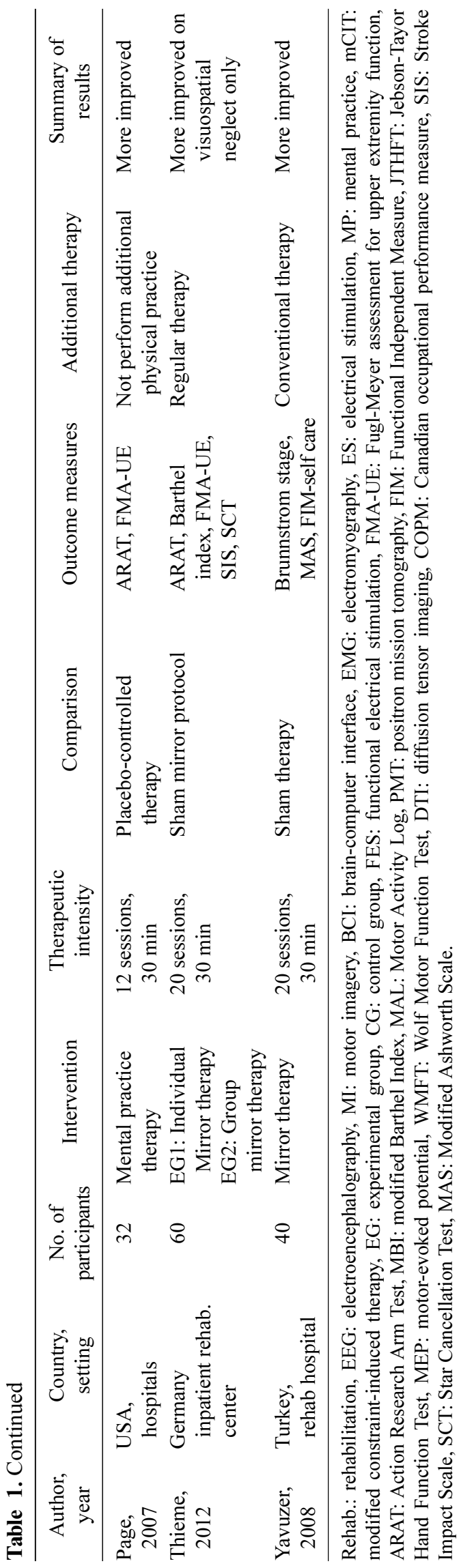

blinding of the outcome assessment $[8,9,23,25]$, and one study had no blinding of the outcome assessment, and the outcome measurement was likely to be influenced by lack of blinding [26]. The other twelve studies reported blinding of the outcome assessment.

\section{Effectiveness of motor imagery on upper limb function in the RCTs}

Nine RCTs involving 256 stroke patients assessed the FMA-UE, and reported improvements in FMA-UE scores after motor imagery training compared with the control group. A fixed-effect model was selected based on the results of significant heterogeneity $(3.43 ; 95 \% \mathrm{CI}, 1.65$ to 5.22; heterogeneity $\left[\mathrm{chi}^{2}=8.03, \mathrm{df}=8, \mathrm{I}^{2}=0 \%\right]$ ), test of overall effect $Z=3.76$; test for subgroup differences $\left[\mathrm{chi}^{2}=2.56\right.$, $\left.\mathrm{df}=2, \mathrm{I}^{2}=21.8 \%\right]$ ) (Figure 2). Four RCTs involving 145 subjects with stroke assessed the ARAT and reported improvements in ARAT scores after motor imagery training compared with the control group. A random-effect model was selected based on the results of significant heterogeneity (1.32; $95 \%$ CI, -8.12 to 10.76 ; heterogeneity $\left[\mathrm{Tau}^{2}=70.74\right.$, $\left.\mathrm{chi}^{2}=15.22, \mathrm{df}=3, \mathrm{I}^{2}=80 \%\right]$, Test of overall effect $\mathrm{Z}=3.76$ ) (Figure 3).

\section{Discussion}

This review was conducted to determine the effects of motor imagery on the recovery of upper limb function in persons affected by stroke. Several studies have reported that the effectiveness of motor imagery training as well as motor imagery with brain-computer interface and motor imagery triggered electrical stimulation on upper extremity function in activities of daily living and functional activities for stroke survivors [14,22,27-29]. They provided several therapeutic types to be conducted with motor imagery to improve upper limb function. We also tried to investigate whether the modes of motor imagery would render therapeutic effects to be different on upper extremity function for stroke or not. Regardless of the training types, motor imagery showed beneficial effectiveness on upper extremity function for stroke survivors based on improved FMA-UE and ARAT scores from the nine RCTs.

Stroke survivors are involved in rehabilitation settings for extended periods of time that causes them to suffer from limited physical functionality of their upper extremity following stroke. In rehabilitation and clinical settings, various therapeutic approaches have been provided to overcome the 


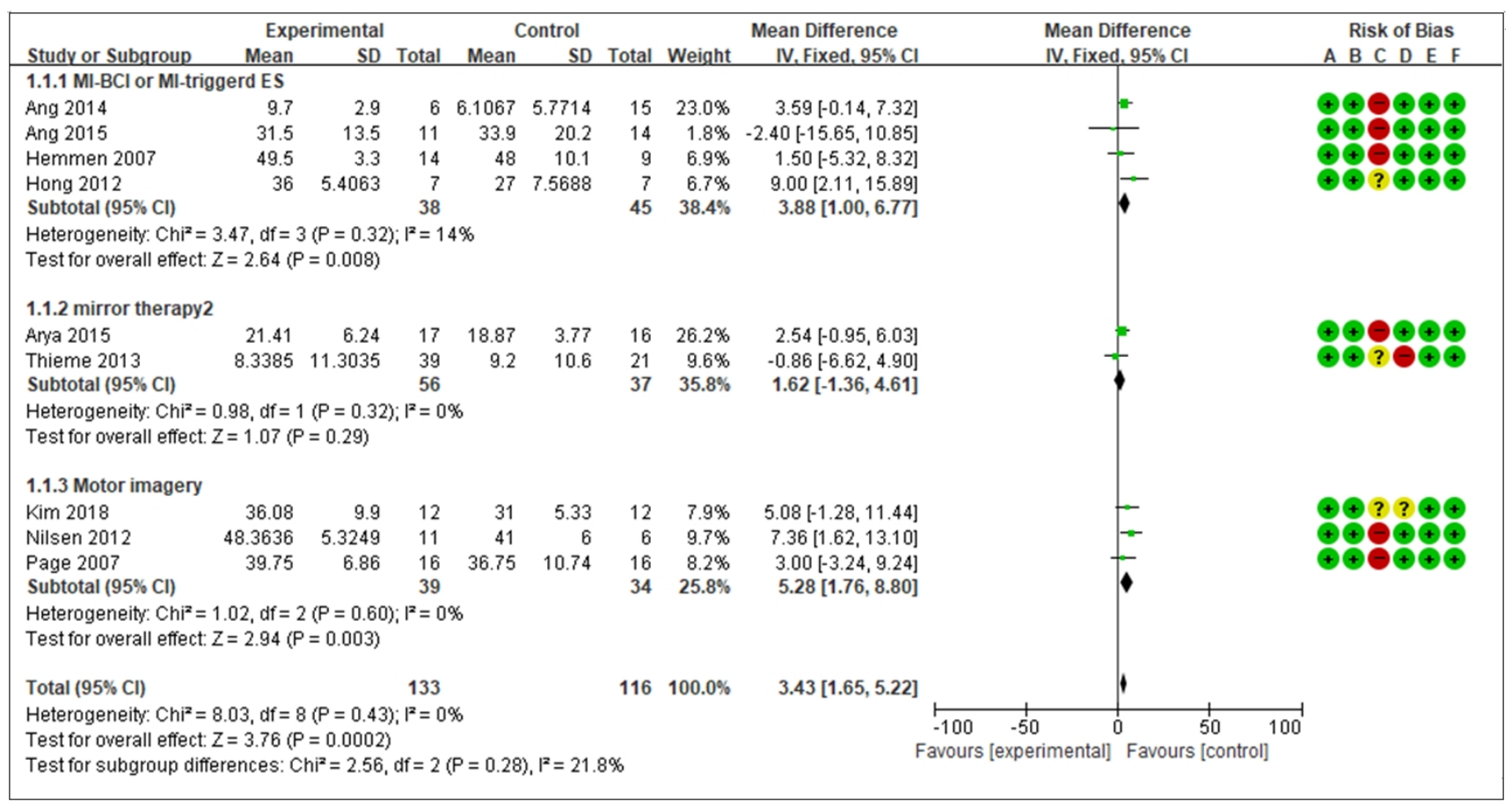

Figure 2. Forest plot of Fugl-Meyer assessment between motor imagery training and control group. A: random sequence generation, B: allocation concealment, C: blinding of participants and personnel, D: blinding of outcome assessment, E: incomplete outcome data, F: selective reporting.

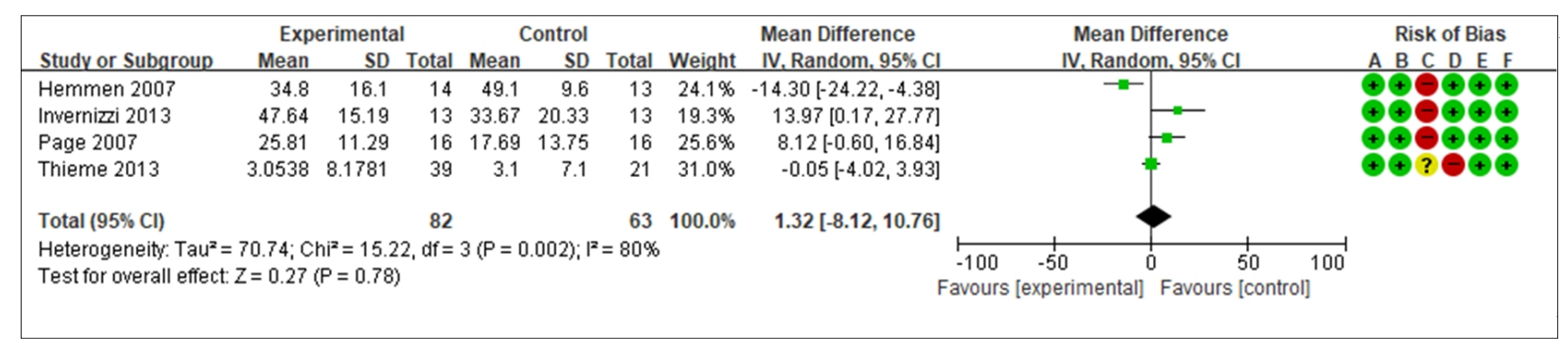

Figure 3. Forest plot of the Action Research Arm Test between the motor imagery training and control group. A: random sequence generation, B: allocation concealment, C: blinding of participants and personnel, D: blinding of outcome assessment, E: incomplete outcome data, F: selective reporting.

limitations in activity performance and participation restrictions due upper limb dysfunction after stroke [8,30-32]. Although these therapeutic approaches have been reported to have feasibility, positive effects, or no treatment effects on upper extremity function for stroke, these approaches have involved repetitive passive or active physical activities and so the participants had some preceding motor function to be able follow the training protocols. Motor imagery of movement is an active, cognitive process used to investigate the content and structure of covert processes that precede the execution of action, and so the training protocol will provide a relatively lower level of mo- tor function or acute stage of the disease following stroke [33-35]. When paired with conventional therapeutic interventions, motor imagery training can be as effective as pure conventional therapeutic interventions for recovery following stroke $[8,14,27,35]$. The results of this review also showed that motor imagery training with other therapeutic interventions based on motor function could exhibit positive effects on upper limb function following stroke. The positive effects after motor imagery training was the same regardless of what type of motor imagery protocol was provided to im- 
prove upper limb function.

This review was conducted to determine the effects of motor imagery training on upper limb function compared to other methods, such as conventional therapy, sham therapy, placebo, and so on following stroke. The review did not consider the type of motor imagery protocol, therapeutic intensity, and post-duration of stroke. Further research will be needed to consider the therapeutic intensity or the duration of treatment after a stroke event. Further studies should also consider when to implement a therapeutic intervention to improve upper limb function for daily and functional activities in persons with stroke in clinical rehabilitation settings.

\section{Conflict of Interest}

The authors declared no potential conflicts of interest with respect to the authorship and/or publication of this article.

\section{References}

1. O'Sullivan SB, Schmitz TJ, Fulk GD. Physical rehabilitation. 6th ed. Philadelphia: F.A. Davis Co.; 2014.

2. Lai SM, Studenski S, Duncan PW, Perera S. Persisting consequences of stroke measured by the Stroke Impact Scale. Stroke 2002;33:1840-4.

3. Bürge E, Kupper D, Finckh A, Ryerson S, Schnider A, Leemann B. Neutral functional realignment orthosis prevents hand pain in patients with subacute stroke: a randomized trial. Arch Phys Med Rehabil 2008;89:1857-62.

4. Page SJ, Dunning K, Hermann V, Leonard A, Levine P. Longer versus shorter mental practice sessions for affected upper extremity movement after stroke: a randomized controlled trial. Clin Rehabil 2011;25:627-37.

5. Page SJ, Levine P, Khoury JC. Modified constraint-induced therapy combined with mental practice: thinking through better motor outcomes. Stroke 2009;40:551-4.

6. Page SJ, Levine P, Leonard A. Mental practice in chronic stroke: results of a randomized, placebo-controlled trial. Stroke 2007; 38:1293-7.

7. Jeannerod M. Neural simulation of action: a unifying mechanism for motor cognition. Neuroimage 2001;14:S103-9.

8. Li F, Zhang T, Li BJ, Zhang W, Zhao J, Song LP. Motor imagery training induces changes in brain neural networks in stroke patients. Neural Regen Res 2018;13:1771-81.

9. Kim SS, Lee BH. Motor imagery training improves upper extremity performance in stroke patients. J Phys Ther Sci 2015; 27:2289-91.

10. Verma R, Arya KN, Garg RK, Singh T. Task-oriented circuit class training program with motor imagery for gait rehabilitation in poststroke patients: a randomized controlled trial. Top Stroke Rehabil 2011;18 Suppl 1:620-32.

11. Liburkina SP, Vasilyev AN, Kaplan AY, Ivanova GE, Chukanova
AS. [Brain-computer interface-based motor imagery training for patients with neurological movement disorders]. Zh Nevrol Psikhiatr Im S S Korsakova 2018;118:63-8.

12. Arvaneh M, Guan C, Ang KK, Ward TE, Chua KSG, Kuah CWK, et al. Facilitating motor imagery-based brain-computer interface for stroke patients using passive movement. Neural Comput Appl 2017;28:3259-72. [Rsuuian]

13. Dodakian L, Campbell Stewart J, Cramer SC. Motor imagery during movement activates the brain more than movement alone after stroke: a pilot study. J Rehabil Med 2014;46:843-8.

14. Okuyama K, Ogura M, Kawakami M, Tsujimoto K, Okada K, Miwa K, et al. Effect of the combination of motor imagery and electrical stimulation on upper extremity motor function in patients with chronic stroke: preliminary results. Ther Adv Neurol Disord 2018;11:1756286418804785.

15. Ang KK, Guan C, Phua KS, Wang C, Zhou L, Tang KY, et al. Brain-computer interface-based robotic end effector system for wrist and hand rehabilitation: results of a three-armed randomized controlled trial for chronic stroke. Front Neuroeng 2014; 7:30.

16. Ang KK, Chua KS, Phua KS, Wang C, Chin ZY, Kuah CW, et al. A randomized controlled trial of EEG-based motor imagery brain-computer interface robotic rehabilitation for stroke. Clin EEG Neurosci 2015;46:310-20.

17. Arya KN, Pandian S, Kumar D, Puri V. Task-based mirror therapy augmenting motor recovery in poststroke hemiparesis: a randomized controlled trial. J Stroke Cerebrovasc Dis 2015;24: 1738-48.

18. Hemmen B, Seelen HA. Effects of movement imagery and electromyography-triggered feedback on arm hand function in stroke patients in the subacute phase. Clin Rehabil 2007;21: 587-94.

19. Invernizzi M, Negrini S, Carda S, Lanzotti L, Cisari C, Baricich A. The value of adding mirror therapy for upper limb motor recovery of subacute stroke patients: a randomized controlled trial. Eur J Phys Rehabil Med 2013;49:311-7.

20. Nilsen DM, Gillen G, DiRusso T, Gordon AM. Effect of imagery perspective on occupational performance after stroke: a randomized controlled trial. Am J Occup Ther 2012;66:320-9.

21. Yavuzer G, Selles R, Sezer N, Sütbeyaz S, Bussmann JB, Köseoğlu F, et al. Mirror therapy improves hand function in subacute stroke: a randomized controlled trial. Arch Phys Med Rehabil 2008;89:393-8.

22. Frolov AA, Mokienko O, Lyukmanov R, Biryukova E, Kotov S, Turbina L, et al. Post-stroke rehabilitation training with a motor-imagery-based brain-computer interface (BCI)-controlled hand exoskeleton: a randomized controlled multicenter trial. Front Neurosci 2017;11:400.

23. Ang KK, Guan C, Chua KS, Ang BT, Kuah C, Wang C, et al. A clinical study of motor imagery-based brain-computer interface for upper limb robotic rehabilitation. Conf Proc IEEE Eng Med Biol Soc 2009;2009:5981-4.

24. Hong IK, Choi JB, Lee JH. Cortical changes after mental imagery training combined with electromyography-triggered electrical stimulation in patients with chronic stroke. Stroke 2012;43: 2506-9.

25. Kim H, Yoo EY, Jung MY, Kim J, Park JH, Kang DH. The effects of mental practice combined with modified constraint-induced 
therapy on corticospinal excitability, movement quality, function, and activities of daily living in persons with stroke. Disabil Rehabil 2018;40:2449-57

26. Thieme H, Bayn M, Wurg M, Zange C, Pohl M, Behrens J. Mirror therapy for patients with severe arm paresis after stroke--a randomized controlled trial. Clin Rehabil 2013;27:314-24.

27. Petersen J, Iversen HK, Puthusserypady S. Motor imagery based Brain Computer Interface paradigm for upper limb stroke rehabilitation. Conf Proc IEEE Eng Med Biol Soc 2018;2018: 1960-3.

28. Prasad G, Herman P, Coyle D, McDonough S, Crosbie J. Applying a brain-computer interface to support motor imagery practice in people with stroke for upper limb recovery: a feasibility study. J Neuroeng Rehabil 2010;7:60

29. Craje C, van der Graaf C, Lem FC, Geurts AC, Steenbergen B. Determining specificity of motor imagery training for upper limb improvement in chronic stroke patients: a training protocol and pilot results. Int J Rehabil Res 2010;33:359-62.

30. Niu CM, Bao Y, Zhuang C, Li S, Wang T, Cui L, et al. Synergy- based FES for post-stroke rehabilitation of upper-limb motor functions. IEEE Trans Neural Syst Rehabil Eng 2019;27:256-64.

31. Huang Q, Wu W, Chen X, Wu B, Wu L, Huang X, et al. Evaluating the effect and mechanism of upper limb motor function recovery induced by immersive virtual-reality-based rehabilitation for subacute stroke subjects: study protocol for a randomized controlled trial. Trials 2019;20:104.

32. Lin LF, Lin YJ, Lin ZH, Chuang LY, Hsu WC, Lin YH. Feasibility and efficacy of wearable devices for upper limb rehabilitation in patients with chronic stroke: a randomized controlled pilot study. Eur J Phys Rehabil Med 2018;54:388-96.

33. Decety J, Ingvar DH. Brain structures participating in mental simulation of motor behavior: a neuropsychological interpretation. Acta Psychol (Amst) 1990;73:13-34.

34. Markman KD, Klein WMP, Suhr JA. Handbook of imagination and mental simulation. New York: Psychology Press; 2009.

35. Zimmermann-Schlatter A, Schuster C, Puhan MA, Siekierka E, Steurer J. Efficacy of motor imagery in post-stroke rehabilitation: a systematic review. J Neuroeng Rehabil 2008;5:8. 